\title{
Real-time Fluorescence Detection of Protein Transduction into Live Cells
}

\author{
Ya-Jung Lee, Silpi Datta, Jean-Philippe Pellois* \\ Department of Biochemistry and Biophysics, Texas A\&M University, College Station, TX \\ 77843, USA
}

\section{Supporting Information}

\section{Experimental Procedures}

All Fmoc amino acid derivatives and resins were purchased from Novabiochem (San Diego, CA). Boc-Cys(StBu)-OH was purchased from Fluka (Milwaukee, WI). The succinimidyl ester of 6-carboxyfluorescein was prepared according to a reported procedure. ${ }^{1}$ All other chemical reagents were purchased from Aldrich Chemical Co (Milwaukee, WI). Analytical gradient reversed-phase HPLC was performed on a HewlettPackard 1200 series instrument and a Vydac C18 column (5 micron, 4 x $150 \mathrm{~mm}$ ). Flow rate was $1 \mathrm{~mL} / \mathrm{min}$ and detection was at 214, 365, 450 and $525 \mathrm{~nm}$. Preparative HPLC was performed on a Vydac C18 $10 \times 250 \mathrm{~mm}$ column. Flow rate was $4 \mathrm{~mL} / \mathrm{min}$ and detection was at 214, 365, 450 and $525 \mathrm{~nm}$. All runs used linear gradients of $0.1 \%$ aqueous TFA (solvent A) and 90\% acetonitrile plus 0.1\% TFA (solvent B). Mass spectrometric analysis was routinely performed on all synthetic peptides. Electrospray mass spectrometry (ESMS) was performed on a Sciex QSTAR spectrometer (Perkin Elmer, Waltham, MA) and MALDI-TOF mass spectrometry was performed on a Kratos Axima CFR (Shimadzu, Columbia, MD). Protein purification was performed on FPLC systems (Amersham Pharmacia Biotech, Piscataway, NJ). Expressed proteins were routinely analyzed by sodium dodecyl sulfate polyacrylamide gel electrophoresis (SDS-PAGE) using standard protocols.

\section{Protein semisynthesis:}

All peptides were prepared using Fmoc solid-phase chemistry on a Rink-amide MBHA resin as previously described. ${ }^{1}$ Fmoc-Lys(Mtt)-OH, Boc-Cys(SStBu)-OH, and 6carboxyfluorescein were used for the synthesis of $\mathbf{1}$. The fluorophore was introduced on the solid-phase after selective deprotection of the Mtt group with 2\% TFA. For the 
synthesis of 4, the TAT peptide was first assembled as Fmoc-Lys(Mtt)-Gly-Arg(Pbf)Lys(Boc)-Lys(Boc)-Arg(Pbf)-Arg(Pbf)-Gln-Arg(Pbf)-Arg(Pbf)-Arg(Pbf)-Gly-Rink amide. The N-terminal lysine was deprotected with $2 \%$ TFA and reacted with Dabcyl Nhydroxysuccinimide (NHS) ester. The N-terminal Fmoc was then removed and the peptide reacted with 3-(2-pyridyldithio)propionic acid NHS ester. Peptides were cleaved from the resin and purified over semi-preparative HPLC. The pure peptides were characterized as the desired products by mass spectrometry analysis $(1$, Observed Mass $=606.4 \mathrm{Da}$, Expected Mass $=606.2 \mathrm{Da} ; 4, \mathrm{OM}=2029.1 \mathrm{Da}, \mathrm{EM}=2028.6 \mathrm{Da})$. The thiol reactive Dabcyl derivative used to prepare protein $\mathbf{6}$ was synthesized by reaction of Dabcyl NHS ester with 2-(pyridyldithio)-ethylamine in methanol. The product, 4-((4(dimethylamino)phenyl)diazenyl)-N-(2-(pyridin-2-yldisulfanyl) ethyl) benzamide was purified by silica gel chromatography and analyzed by mass spectrometry $(\mathrm{OM}=438.2 \mathrm{Da}$, $\mathrm{EM}=438.2 \mathrm{Da})$

Recombinant mCherry was obtained by subcloning the mCherry gene in a pTXB1-EGFP previously cloned. ${ }^{1}$ The DNA encoding mCherry was isolated by PCR from the pRSET-B mCherry plasmid (provided by R.Y. Tsien, HHMI investigator, Department of Pharmacology, UCSD, CA) using the 5' primer (5'-GGC GAC CAT ATG GTG AGC AAG GGC GAG GAG-3') and 3' primer (5'-GCC GCC GAA TTC CTT GTA CAG CTC GTC CAT GCC-3'), encoding the NdeI and EcoRI restriction sites, respectively. The digested PCR product was ligated into the NdeI,EcoRI digested pTXB1-EGFP vector. This cloning procedure results in the insertion of the amino acid sequence EFGKKKRKVG between the C-terminal lysine of mCherry and the $\mathrm{N}$-terminal cysteine of Mxe GyrA. The protein was expressed and purified according to previously established protocols.

The ligation reaction was performed at $4^{\circ} \mathrm{C}$ by adding the chemically synthesized peptide 1 to the mCherry thioester solution $(300 \mu \mathrm{M})$ to a final concentration of $1 \mathrm{mM}$ (Figure S1 and S2). The ligated protein was purified from the excess peptide by gel filtration chromatography (Superdex 200, Amersham Biosciences) in a buffer of $25 \mathrm{mM}$ Hepes, 100 $\mathrm{mM} \mathrm{NaCl}, 100 \mathrm{mM}$ 1,6-hexanediol, $\mathrm{pH}$ 7.5. The ligated protein was then allowed to dimerize by Cys to Cys disulfide bond formation overnight at room temperature. The protein dimer was purified from monomeric species (consisting principally of unligated material) by a second step of gel filtration chromatography. The purified dimer was then reduced to generate a pure ligated and monomeric product by incubation with immobilized TCEP disulfide reducing gel (Pierce, Rockford, IL, used according to the manufacturer's protocol). The freshly reduced proteins $(20 \mu \mathrm{M})$ were incubated with peptide $4(30 \mu \mathrm{M})$ 
for $10 \mathrm{~min}$ or Dabcyl 2-(pyridyldithio)-ethylamine (30 $\mu \mathrm{M})$. The products were analyzed by SDS-PAGE (Figure S3). The proteins $\mathbf{5}$ and $\mathbf{6}$ were purified from the excess labeling reagents by gel filtration chromatography (Superdex 200, Amersham Biosciences) in a buffer of $25 \mathrm{mM}$ Hepes, $100 \mathrm{mM} \mathrm{NaCl}, 100 \mathrm{mM}$ 1,6-hexanediol, $\mathrm{pH}$ 7.5. The proteins identities were confirmed by mass spectrometric analysis. (3: $\mathrm{OM}=28317 \pm 4 \mathrm{Da}, \mathrm{EM}=$ $28313 \mathrm{Da}$ after loss of the N-terminal Methionine; 5: $\mathrm{OM}=30240 \pm 10 \mathrm{Da}, \mathrm{EM}=30232$ $\mathrm{Da} ; 6$ : $\mathrm{OM}=28636 \pm 10 \mathrm{Da}, \mathrm{EM}=28640 \mathrm{Da})$.

\section{Fluorescence Spectroscopy:}

Experiments were conducted at room temperature in a stirred $1 \mathrm{~cm}$-pathlength cuvette using a Koala spectrofluorometer (ISS, Champagne, IL). Excitation was at $488 \mathrm{~nm}$ with a $0.5 \mathrm{~mm}$ slit and the fluorescence emission was monitored from 498 to $700 \mathrm{~nm}$ through a $0.5 \mathrm{~mm}$ slit. The protein was diluted to concentrations ranging from 100 to $1 \mu \mathrm{M}$ in a buffer containing $200 \mathrm{mM} \mathrm{NaCl}, 100 \mathrm{mM}$ Hepes buffer (pH 7.5).

\section{Live-cell Imaging Experiments:}

HeLa cells were cultured on $35 \mathrm{~mm}$ plates (P35G-1.5-7-C-grid, MatTek Corp., Ashland, MA) at 100,000 cells $/ \mathrm{mL}$ in a total volume of $2 \mathrm{~mL}$ of Dulbecco's modified Eagle's medium supplemented with $10 \%$ FBS in a humidified atmosphere containing $5 \% \mathrm{CO}_{2}$. For live-cell imaging, cells were washed and incubated with Leibovitz's L-15 Medium lacking L-cysteine and placed under a spinning disk confocal microscope (Axiovert 200M, Carl Zeiss, with a PerkinElmer UltraView LCI system) equipped with a chamber maintained at $37^{\circ} \mathrm{C}$. Cells were microinjected using a commercial microinjection system (Femtojet, Eppendorf, Westbury, NY). Images were collected with 2 x 2 binning using a Hamamatsu ORCA-ER digital CCD camera mounted on the microscope with an apo63× / 1.4 NA oil objective. Images were acquired using three different filter sets: $E x=488 \mathrm{~nm} / E m=525 \mathrm{~nm}$ (donor image), $E x=568 \mathrm{~nm} / \mathrm{Em}=620 \mathrm{~nm}$ (acceptor image), and $E x=488 \mathrm{~nm} / E m=620 \mathrm{~nm}$ (FRET image) (Figure S4). Cells microinjected or incubated with 3, 5, or $\mathbf{6}$ were imaged $20 \mathrm{sec}$ after microinjection or addition of the protein to the media and for up to $3 \mathrm{~h}$. In order to provide comparable data, image acquisition was performed using the same conditions throughout the experiments. In particular, detection of donor, acceptor, and FRET signals was done with $500 \mathrm{~ms}$ or $1000 \mathrm{~ms}$ excitation for the 3 channels. 
Fluorescence properties of the transduction probe microinjected in the cytosol of live cells:

In order to establish what the fluorescence properties of our transduction probe might be after translocation, 1, 2, and $\mathbf{3}$ were directly microinjected into the cytosol of human cervix carcinoma cells (HeLa). Cells were imaged for up to $3 \mathrm{~h}$ after microinjection with a spinning disk confocal microscope. Images were acquired using three different filter sets: $\mathrm{Ex}=488 \mathrm{~nm} / \mathrm{Em}=525 \mathrm{~nm}$ (donor image, Fluorescein), $\mathrm{Ex}=568 \mathrm{~nm} / \mathrm{Em}=620 \mathrm{~nm}$ (acceptor image, mCherry), and Ex=488nm/Em=620nm (FRET image). The morphology of the microinjected cells did not change over that period of time and the intracellular fluorescence intensities measured at the different time points were constant. This indicates that the proteins were not cytotoxic and that they were not significantly degraded in the cytosol within this experimental time frame. The measured FRET intensity from the FRET filter set was corrected for the cross talk from the donor and acceptor (determined by microinjection of $\mathbf{1}$ and $\mathbf{2}$ alone). ${ }^{2}$ Microinjected $\mathbf{3}$ was uniformly distributed in the cytosolic and nuclear compartments and showed a strong FRET signal with Donor to FRET and Acceptor to FRET ratios of 2.0 +/- 0.3 and 1.6 +/- 0.2, respectively (Figure S5).

Images were acquired using three different filter sets: $E x=488 \mathrm{~nm} / E m=525 \mathrm{~nm}$ (donor image), $\mathrm{Ex}=568 \mathrm{~nm} / \mathrm{Em}=620 \mathrm{~nm}$ (acceptor image), and $\mathrm{Ex}=488 \mathrm{~nm} / \mathrm{Em}=620 \mathrm{~nm} \quad(\mathrm{FRET}$ image). FRET was determined using the metamorph software (version 6.0, Universal Imaging Corp.,Downingtown, PA ) and correction methods according to:

$\mathrm{F}=\left(\right.$ fret $\left._{\mathrm{gv}}-\mathrm{bg}_{\mathrm{fret}}\right)-\mathrm{cf}_{\mathrm{don}} *\left(\mathrm{don}_{\mathrm{gv}}-\mathrm{bg}_{\mathrm{don}}\right)-\mathrm{cf}_{\mathrm{acc}} *\left(\mathrm{acc}_{\mathrm{gv}}-\mathrm{bg}_{\mathrm{acc}}\right)$

where

$\mathrm{F}=$ FRET intensity

$\mathrm{gv}=$ intensity as gray value

bg $=$ background intensity

$\mathrm{cf}=$ correction factor

fret $=$ FRET image

don $=$ donor image

acc $=$ acceptor image

The measured FRET intensity from the FRET filter set is corrected for the cross talk from the donor alone and acceptor alone. Correction factors for crosstalk correction were determined to be $\mathrm{cfdon}=0.125$ and $\mathrm{cfacc}=0.098$ (calculated after microinjection of 1 and 2 alone, Figure S5).

After image correction, FRET intensities averaged over a region of interest (ROI) were measured using the metamorph software. Mean intensities and their corresponding 
standard deviations were recorded for ROI drawn over nuclear and cytoplasmic areas of the cell.

\section{Fluorescence Activation of 5 in the cytosol of live cells:}

To determine whether 5 would be reduced by cellular GSH, we microinjected the protein into the cytosol of live cells and measured the increase in donor and FRET signals that result from loss of quenching. Approximately $60 \%$ of the donor was unquenched within 20 seconds after microinjection and disulfide bond cleavage was complete within 2 minutes (Figure S6). Donor fluorescence and FRET increased at the same rate while the fluorescence of the mCherry acceptor was constant throughout the experiment. Interestingly, the final Donor to FRET and Acceptor to FRET ratios of unquenched $\mathbf{5}$ were similar to those of microinjected 3. Overall, we concluded that the rapid kinetics of fluorescence activation was compatible with real-time imaging and that the observed fluorescence ratios were specific to a homogenous population of reduced protein.

\section{Monitoring protein transduction into HeLa cells:}

The internalization of TAT-protein conjugates has previously been reported to involve macropinocytosis. ${ }^{3}$ A key question was therefore to determine whether the fluorescence of our probe would be activated in endocytic compartments. In order to determine whether protein 5 might undergo reduction in endosomes, the cysteine of protein $\mathbf{3}$ was labeled with a 2-pyridyldithio-Dabcyl derivative to generate protein $\mathbf{6}$, a protein with similar fluorescence properties as protein $\mathbf{5}$ but lacking the TAT peptide. (Figure S2). HeLa were incubated with a $1 \mu \mathrm{M}$ solution of $\mathbf{3 , 6}$, or $\mathbf{5}$, washed after 1 hour and analyzed by confocal fluorescence microscopy (Figure S7). Cells incubated with 3 and $\mathbf{6}$ contained internalized material with only a punctated distribution (similar to that of markers of fluid-phase endocytosis such as $70 \mathrm{kDa}$ Dextran-Oregon green 448. 6 and dextran Oregon green were incubated together with live cells and found to be internalized in identical endocytic compartments, as demonstrated by the colocalization of the red (from 6) and green (from dextran oregon green) signals, data not shown). Protein $\mathbf{3}$ was used as a control that would mimic the product obtained from endocytic reduction of protein 6 . Cells incubated with 3 showed donor, acceptor, and FRET fluorescence. This demonstrates that fluorescein, a $\mathrm{pH}$ sensitive fluorophore, could still be detected even if its fluorescence might be reduced at the acidic $\mathrm{pH}$ of the endocytic compartments detected. In contrast, cells incubated with 6 showed only acceptor fluorescence, indicating that no disulfide bond cleavage had occurred during the 1 hour period. 


\section{Analysis of punctate versus diffuse fluorescence:}

Images were analyzed with the Metamorph software. To analyze and compare the fraction of protein present in endocytic vesicles (acidic $\mathrm{pH} \approx 5$ ) from the fraction released into the cytosol $(\mathrm{pH} \approx 7)$, we used the acceptor images (mCherry fluorescence), as the emission intensity of mCherry is constant between $\mathrm{pH} 5$ and $\mathrm{pH} 7$ (as determined by in vitro fluorescence characterization, data not shown). It is difficult to identify a punctate object if its fluorescence intensity is similar the diffuse fluorescence in the cells. We therefore focused our analysis on cells that had a low diffuse fluorescence as they appeared to have the greatest contrast between punctate and diffuse fluorescence. The cells were first identified and separated as objects by morphometry analysis and the total fluorescence intensity within each cell was then measured (this includes both punctate and diffuse fluorescence and represents the summed fluorescence over all pixels). The objects of punctuate fluorescence within each cell were identified and separated using fluorescence intensity thresholds. Objects with a fluorescence intensity greater than the local diffuse fluorescence (the fluorescence surrounding the bright object) by more than 2-fold were assigned as punctate fluorescence objects (all objects identified with this threshold were indeed punctate and consistent with endocytic compartments). The fluorescence intensities of these objects were summed and the ratios of the fluorescence intensities of punctate objects/whole cells were then measured. In the acceptor image displayed in Figure 2B (60 min incubation, the brightest cell could not be analyzed), the calculated punctate to total fluorescence ratios ranged from $50 \%$ to $90 \%$.

\section{Quantitative evaluation of intact and degraded protein populations after transduction into HeLa cells:}

Different populations of protein might be present in the cytosol or nucleus of cells after the transduction of protein 5 (Figure S9). In particular, the protein can either successfully reach its destination while remaining intact or become degraded during the translocation process. As a matter of fact, green fluorescence is independent from the biophysical state of the protein and can be expected from not only the intact protein but from any degradation products as well. The FRET signal however can only be detected if the red fluorescent protein is folded and intramolecularly linked to the green fluorophore. In principle, this signal cannot account for partial proteolysis of the protein at a site Nterminal of the protein chromophore (residue $70-72$ in a protein sequence of 248 amino acids) that would preserve protein red fluorescence and linkage to the C-terminal green fluorophore. However, proteolysis between the protein's chromophore and the green label 
as well as protein denaturation would result in the suppression of the FRET signal. The FRET signal therefore provides a specific signal that can validate the successful delivery of a population of relatively intact protein (at worse proteolyzed at the N-terminus, yet still folded and fluorescent). In contrast, the green fluorescence signal results from the contributions of both intact and degraded protein populations. The contribution of the intact protein in the green channel can however be obtained directly from the FRET signal with the donor to FRET ratio established from the microinjected 3. As a result, the contribution of the degraded protein population can be evaluated by substracting the signal of the intact protein in the green channel from the total green signal.

To quantitatively analyze the various protein populations generated during the translocation process, the relative intensities from the donor, acceptor, and FRET images of cells incubated with protein $\mathbf{5}$ for 1 hour were analyzed and compared to that of cells microinjected with 5. The fluorescence intensities in the donor, acceptor, and FRET images were integrated over the nuclear area so as to minimize the contribution of endocytic compartments in the acceptor image. Significant increases in the donor to FRET and acceptor to FRET ratios were observed in cells incubated with $\mathbf{5}$ when compared to cells microinjected with 3 or $\mathbf{5}$ (Figure S8). Because they represent a loss of FRET between donor and acceptor, these increased ratios are indicative of significant protein denaturation and/or degradation (Figure 2A). In the cells represented in Figure 2B, up to $73 \%$ of the donor signal was attributed to degradation products while only $27 \%$ was attributed to the intact cytosolic protein. When comparing these signals, one has however to take into account the difference in brightness between the green fluorophore that is involved in FRET and the green fluorophore that is not (as measured by the FRET efficiency E). By using a FRET efficiency of $35 \%$ as determined in vitro, the donor signal would suggest that approximately $63 \%$ of the protein in this cell is degraded while $37 \%$ remains intact. After repeating this operation on a population of one hundred cells, we found that on average, $55 \% \pm 10 \%$ of the protein is degraded. Because no significant protein degradation was observed in cells microinjected with $\mathbf{3}$ or $\mathbf{5}$ over the same time period, these results suggest that it is the protein transduction process itself that causes the unfolding or degradation of the protein.

To measure the relative contribution of (the donor that can undergo FRET (i) and and the donor that does not $(\mathrm{d})$ in the donor image, we used the following formula: $\mathrm{F}_{\mathrm{d}+\mathrm{p}}=$ (don$\left.\mathrm{F}_{\mathrm{i}}\right) *(1-\mathrm{E})=($ don- $2.0 *$ fret $) *(1-\mathrm{E})$, where $\mathrm{F}_{\mathrm{d}+\mathrm{p}}$ is the fraction of protein that does not undergo FRET, $F_{i}$ the fraction that can, don is the intensity from the donor image, fret the intensity from the FRET image, and E the FRET efficiency. This formula is based on the fact that $F_{i}$ 
$=2.0 *$ fret in the donor imagefor the microinjected controls $\mathbf{3}$ and $\mathbf{5}$ and that the brightness of fluorescein is 35\% increased (according to the FRET efficiency) upon loss of FRET.

The fraction $\mathrm{F}_{\mathrm{p}}$ of proteolyzed protein $\mathrm{p}$ (which represents species where cleavage has occurred between mCherry and fluorescein while maintaining mCherry fluorescence, as described in Figure S9) present in the cytosol can also be estimated by comparing the acceptor image and FRET image and using the formula $\mathrm{F}_{\mathrm{p}}=$ acc $-1.6 *$ fret, where acc is the diffuse mCherry fluorescence intensity in the acceptor image. This formula is based on the fact that $\mathrm{F}_{\mathrm{i}}=1.6 *$ fret in the acceptor image for the microinjected controls $\mathbf{3}$ and $\mathbf{5}$ (after reduction).

1 J. P. Pellois, T. W. Muir, Angew. Chem. Int. Ed. Engl. 2005, 44, 5713.

2 D. C.Youvan, C. M. Silva, E. J. Bylina, W. J. Coleman, M. R. Dilworth, M. M. Yang, Biotechnology $1997,3,1$.

3 J. S. Wadia, R. V. Stan, S. F. Dowdy, Nat. Med. 2004, 10, 310. 


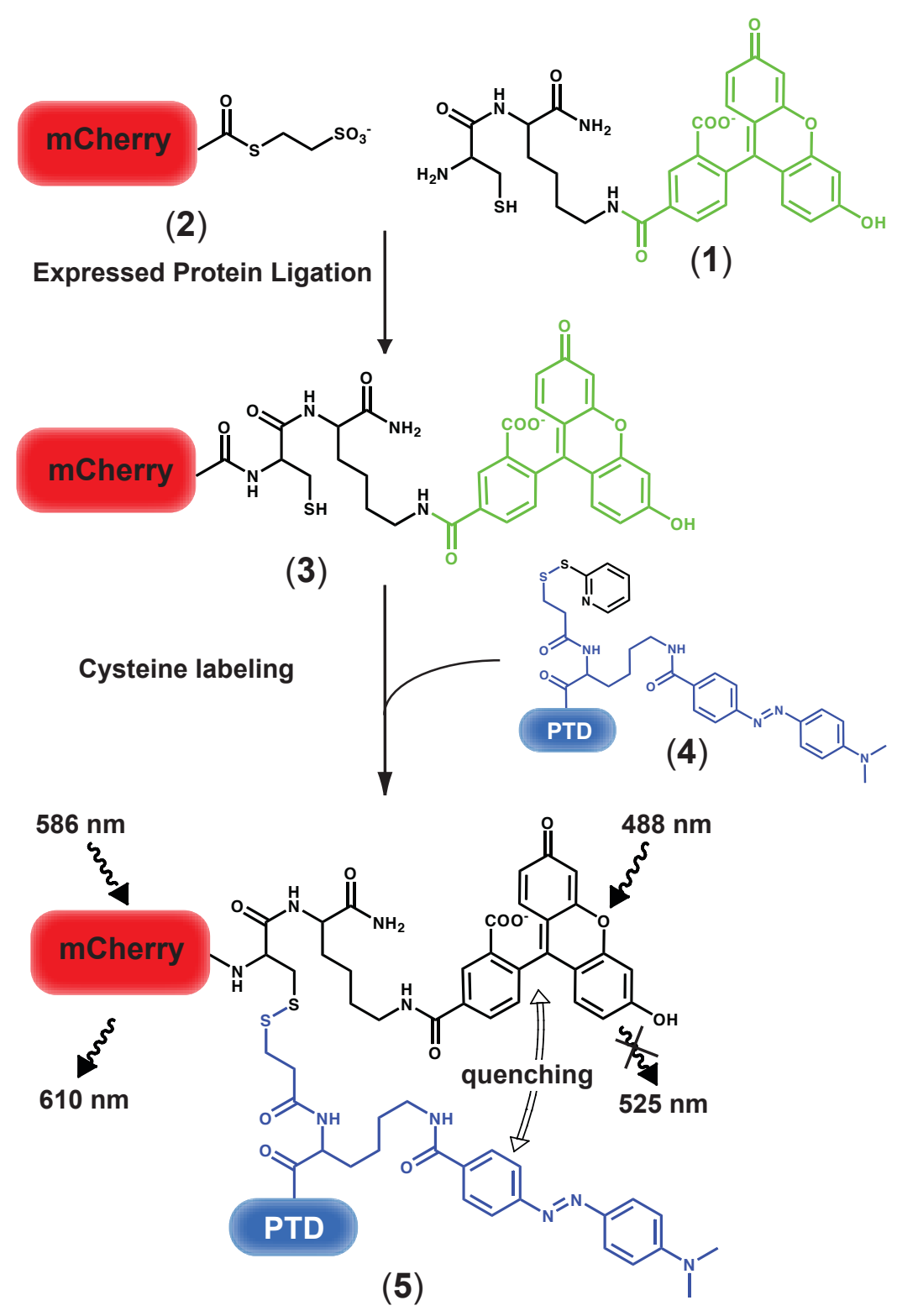

Figure S1: Design and synthesis of a green and red fluorescent protein containing a releasable quencher/PTD conjugate. In our experiments, the protein acceptor is mCherry $(28 \mathrm{kDa})$, the donor carboxyfluorescein, and the quencher Dabcyl. PTD is the HIV TAT peptide GRKKRRQRRR. Protein 5 was assembled from the recombinant mCherry-thioester 2 and two peptides obtained by solid-phase peptide synthesis $(1,4)$. Expressed protein ligation was used to generate a dually fluorescent mCherry which contains a single cysteine (3). Disulfide bond formation between 3 and the 2-pyridyl thiol-activated peptide 4 yields 5 . 


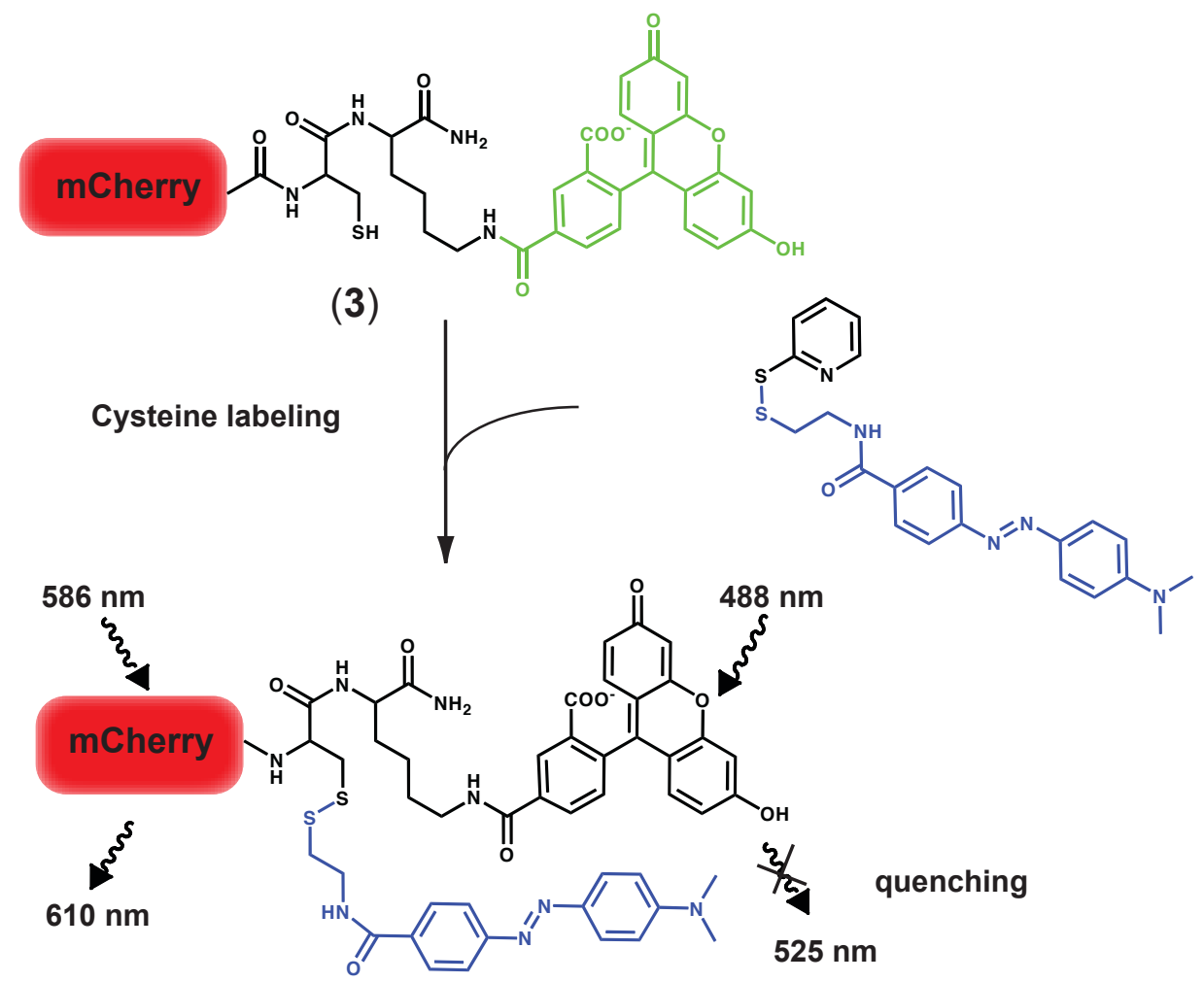

(6)

Figure S2: Design and synthesis of (6), a green and red fluorescent protein containing a releasable quencher conjugate. 


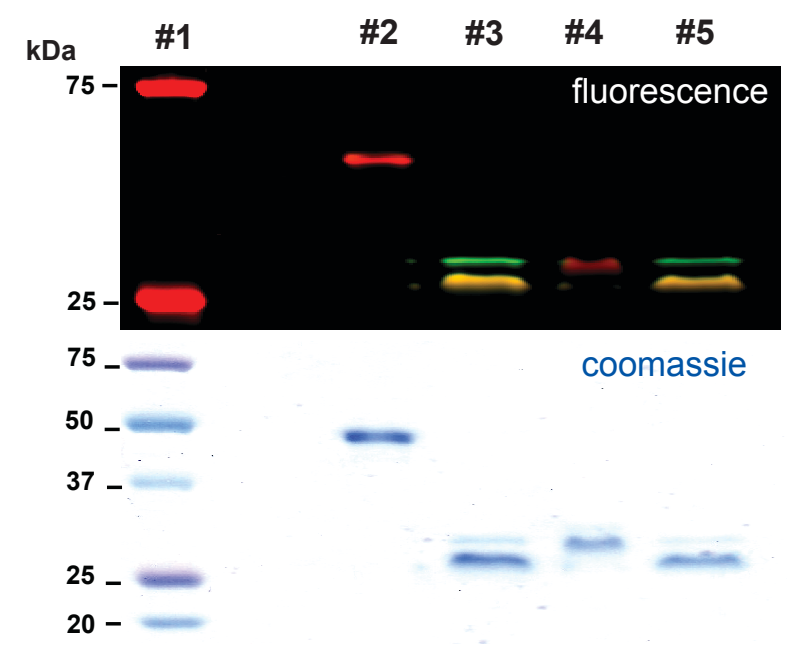

Figure S3: SDS-PAGE analysis of the fluorescent constructs.

Detection was performed with a fluorescent scanner (overlay of green and red emission) or coomassie staining. Lane \#1: Markers; lane \#2: 3 after oxidation; lane \#3: 3 after reduction with iTCEP; lane \#4: 5; lane \#5: 5 after reduction with GSH (the green fluorescent bands in lane \#3 and \#5 are the result of partial protein unfolding during SDS-PAGE analysis). 


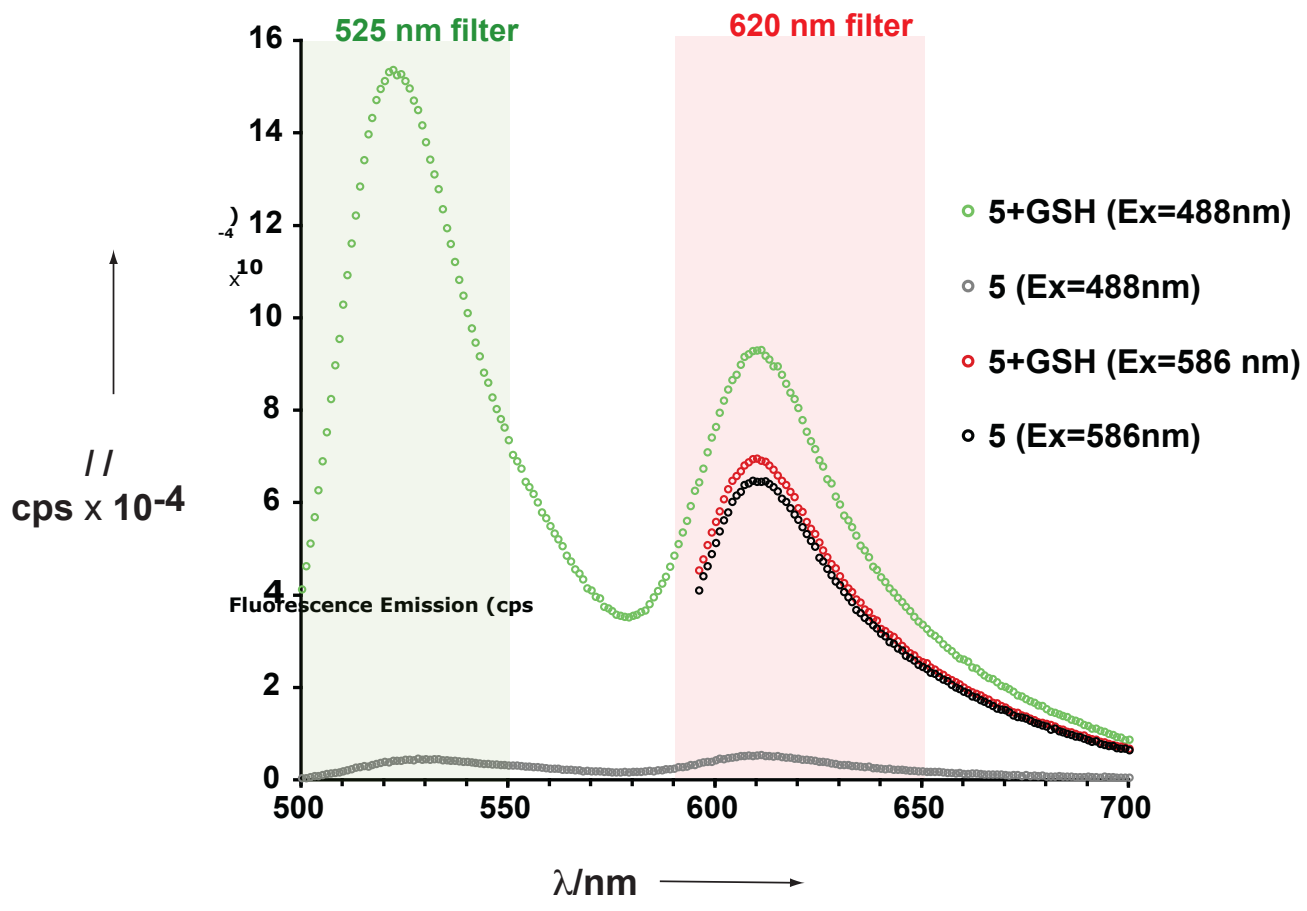

Figure S4: Fluorescence spectra of protein $5(1 \mu \mathrm{M})$ before and 1 min after reduction with GSH (5 mM). Fluorescence excitation was performed at either 488 or $586 \mathrm{~nm}$.

The characteristics of the emission filters used for fluorescence microscopy are indicated. 
microinjected peptide or protein:

(1)

(2)

(3)

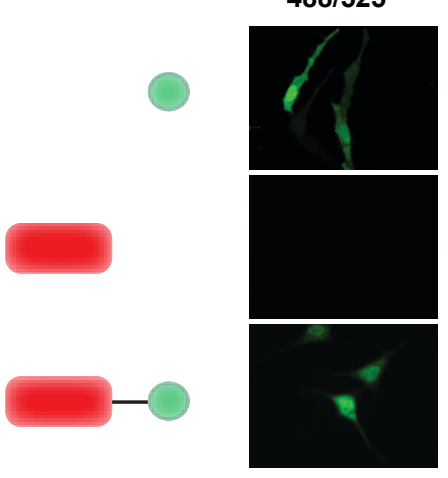

$$
\begin{aligned}
& \text { FRET }=\text { Raw FRET }-A^{*} \text { Donor }-B^{*} \text { Acceptor } \\
& \text { FRET }=(488 / 620)-A^{*}(488 / 525)-B^{*}(568 / 620)
\end{aligned}
$$

Figure S5. Microinjection of 1, 2, and 3 into HeLa cells for the determination of filter cross talk from the donor and acceptor alone and FRET measurement. 
(A)

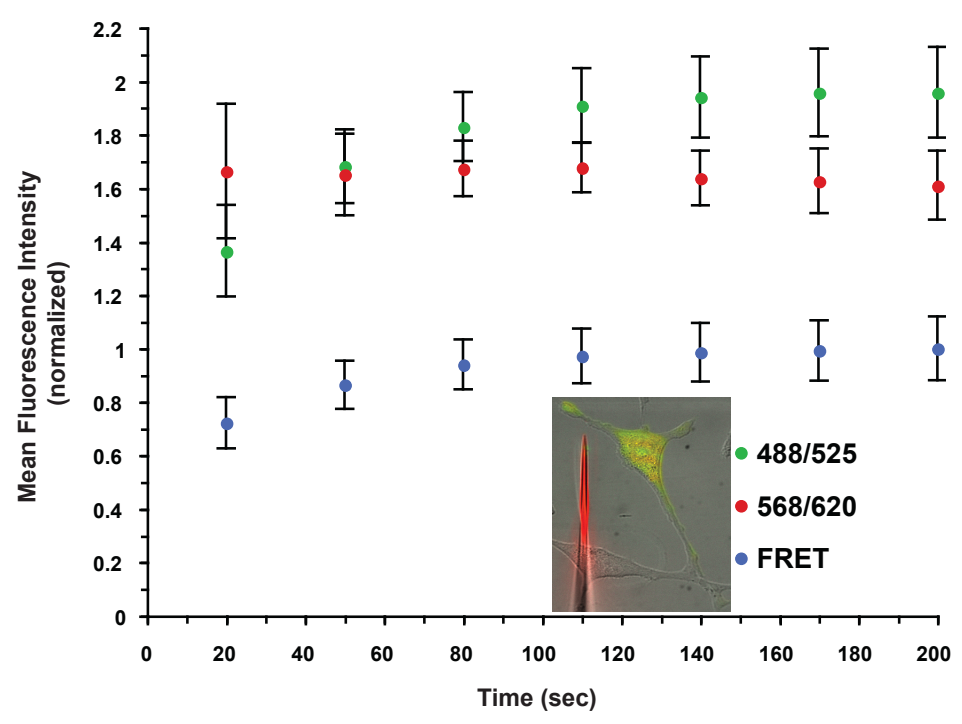

(B)

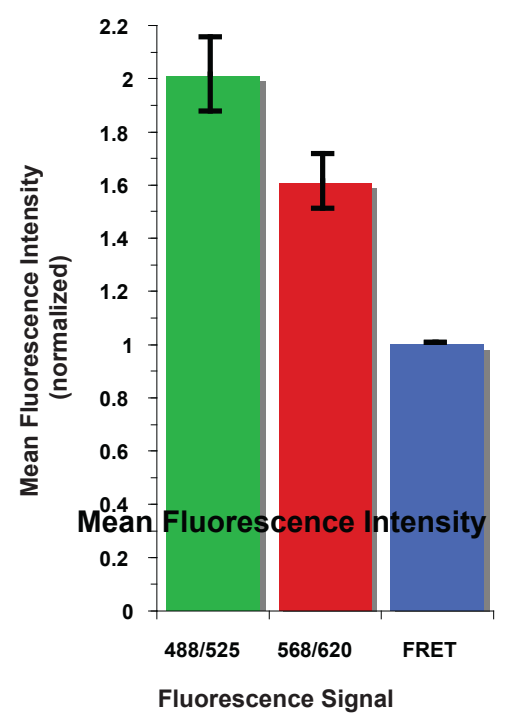

Figure S6: Microinjection of 5 and 3 for the determination of the rate of fluorescence activation in the cytosol of HeLa cells and characterization of the Donor/FRET and Acceptor/FRET ratios. (A) Plot representing the relative donor (488/525), acceptor (568/620), and FRET fluorescence intensities averaged over 12 cells microinjected with 5 . Images were recorded as early as $20 \mathrm{sec}$ after microinjection. The intensities are normalized to the FRET signal at the $200 \mathrm{sec}$ time point.

(B) Relative donor, acceptor, and FRET fluorescence intensities averaged over 20 cells microinjected with 3 . The intensities are normalized to the FRET signal. 
Donor:
$E x=488 \mathrm{~nm}$
$E m=525 \mathrm{~nm}$

Acceptor:
Ex $=568 \mathrm{~nm}$
$E m=620 \mathrm{~nm}$

FRET:

$E x=488 \mathrm{~nm}$

$\mathrm{Em}=620 \mathrm{~nm}$

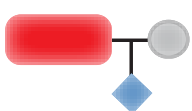

(6)

(after 60 min of incubation)

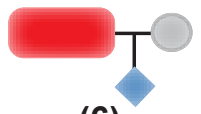

(6)
Donor/Acceptor overlay

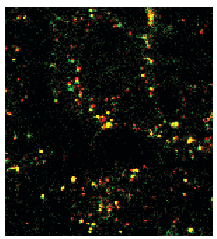

(after $60 \mathrm{~min}$ of incubation)

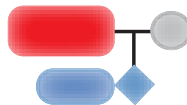

(5)

(after $10 \mathrm{~min}$ of incubation)

70 kD Dextran-Oregon green +

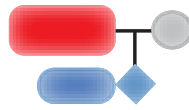

(5)

(after $10 \mathrm{~min}$ of incubation)

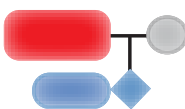

(5)

(after $60 \mathrm{~min}$ of incubation)
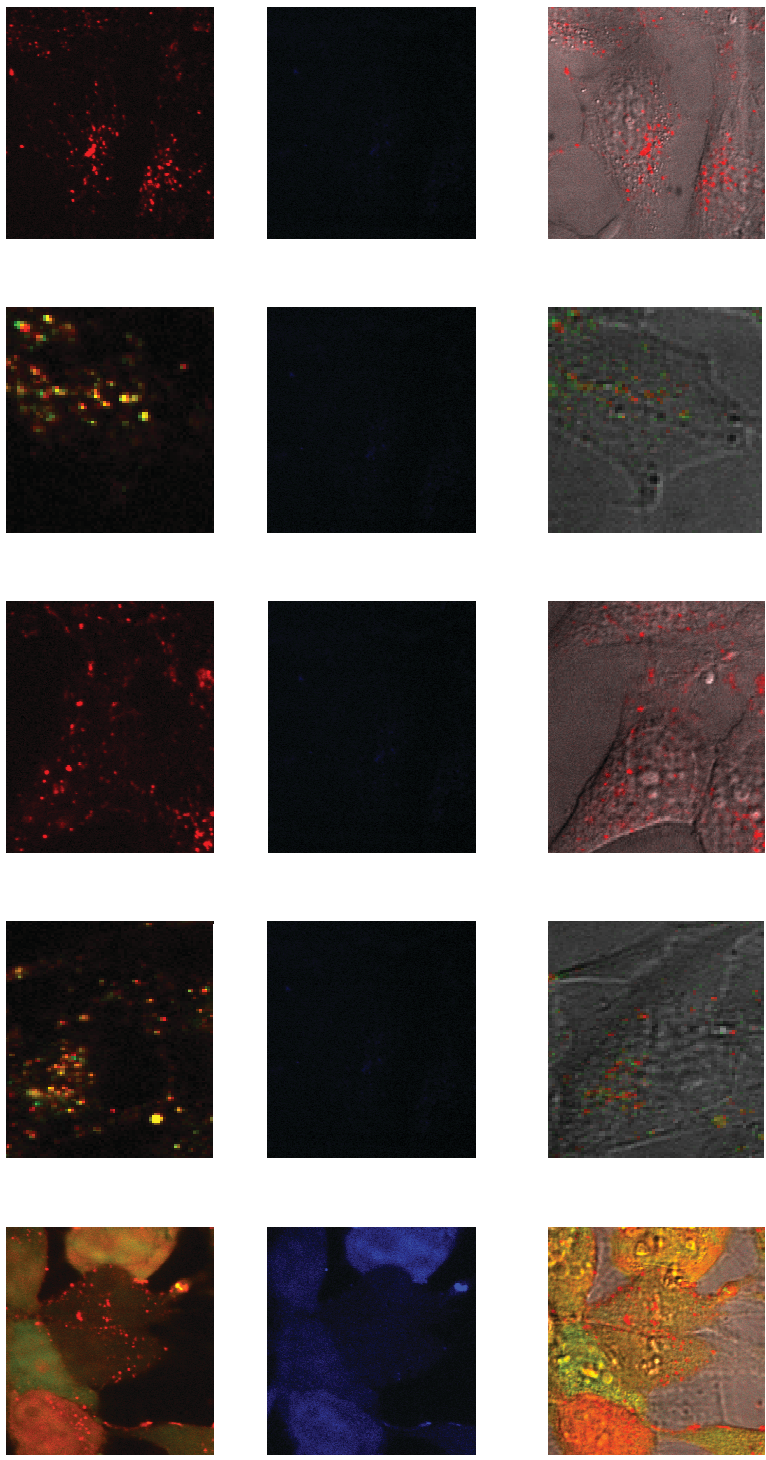

Figure S7: Representative fluorescence images from cells incubated with proteins 3, 6, and 5 with without the fluid-phase endocytosis marker $70 \mathrm{kD}$ Dextran-Oregon green (oregon green has a fluorescence emission spectra similar to fluorescein and is detected in the donor channel).

Cells were incubated with $1 \mu \mathrm{M}$ protein solutions in media lacking L-cysteine and imaged after 10 or $60 \mathrm{~min}$. The donor/acceptor overlay (in green and red pseudo-color, respectively), the FRET image (in blue pseudo-color), and the donor/acceptor/DIC overlay are represented. Cells incubated with 3 and 6 contained internalized material with only a punctate distribution. While cells incubated with 3 showed donor, acceptor, and FRET fluorescence, cells incubated with 6 showed only acceptor fluorescence, indicating that disulfide bond cleavage did not occur at a level sufficient to be detected by donor and FRET fluorescence.

When incubated with $70 \mathrm{kD}$ Dextran-Oregon green, the punctate acceptor fluorescence from both 5 or 6 colocalized with the oregon green fluorescence, suggesting that 5 and 6 are both internalized by fluid-phase endocytosis (colocalization is characterized by the yellow color resulting from the overlay from the green and red images; not all endocytic vesicles are yellow because of differences in the green and red intensities as well as movement of the vesicles during image acquisition). 
(A)

Donor image

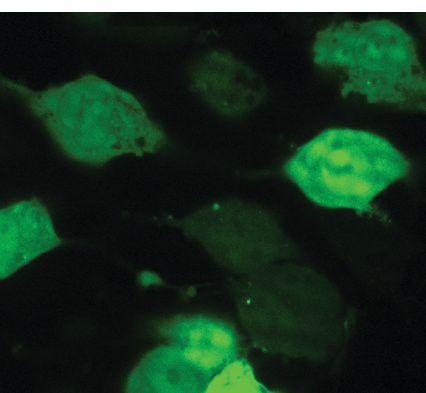

(B)

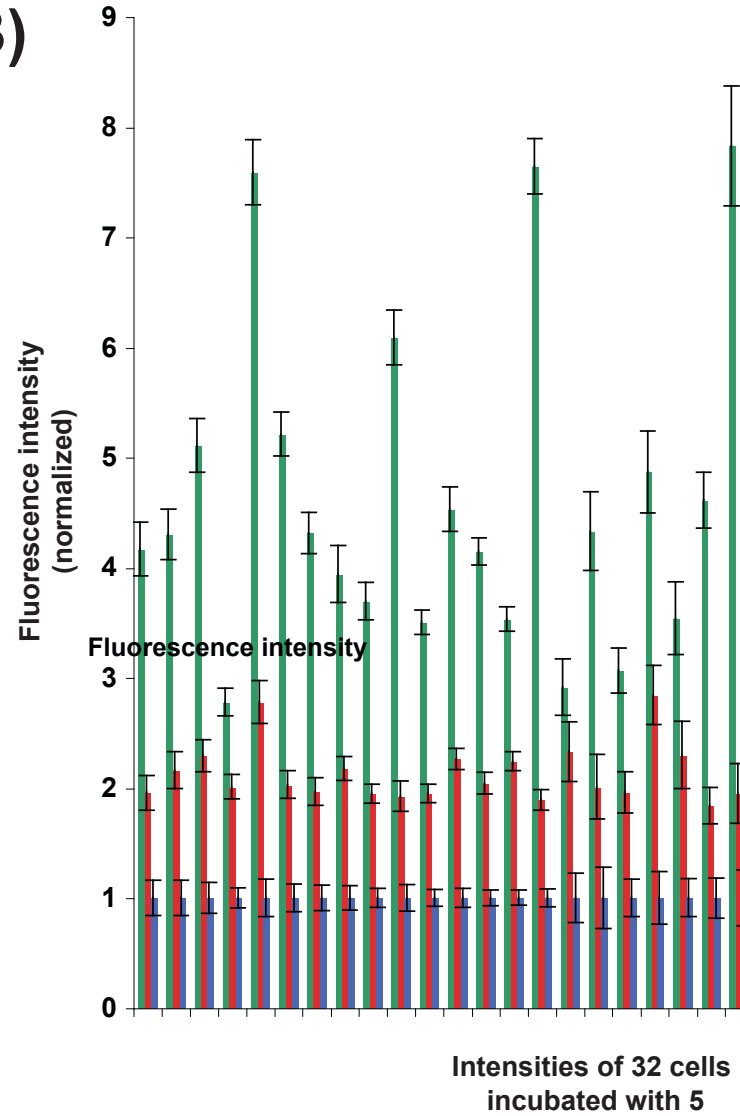

Acceptor image
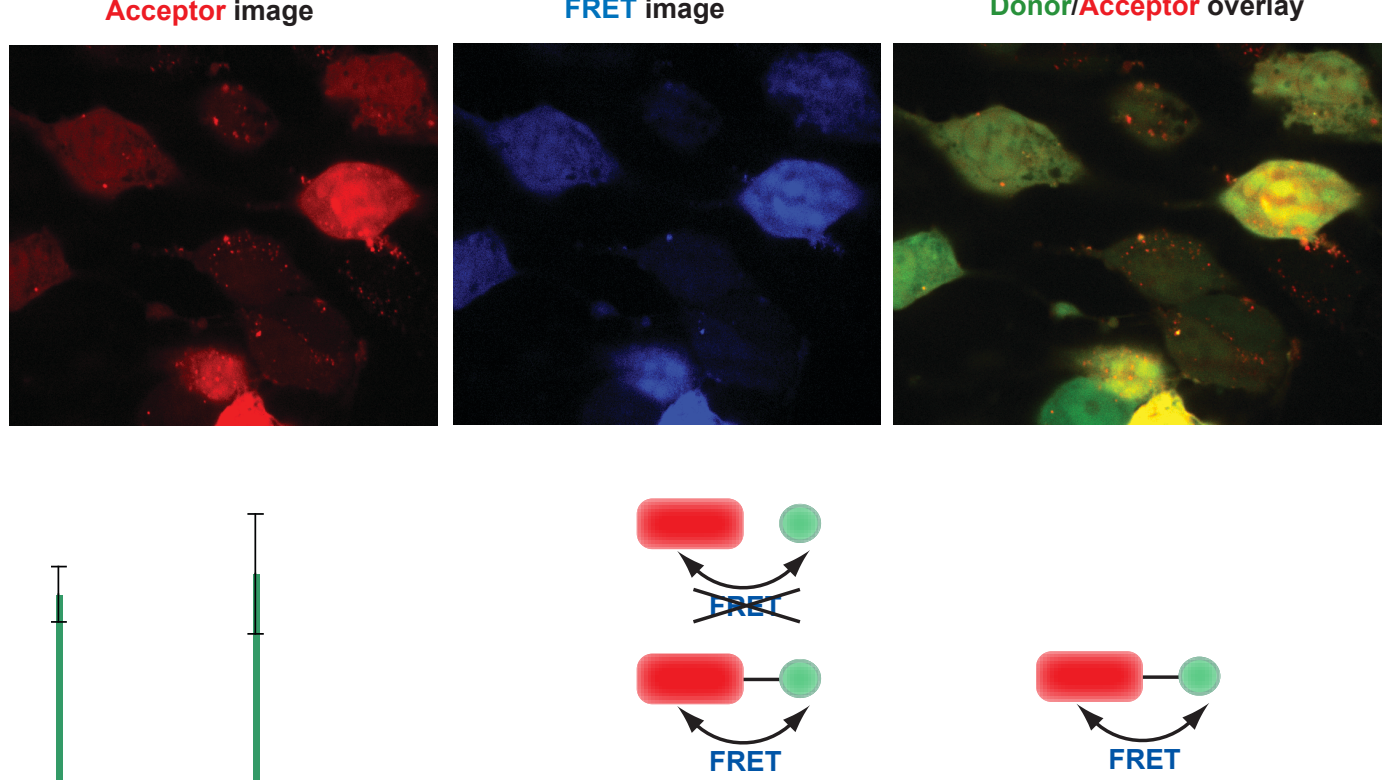

FRET

Acceptor

FRET

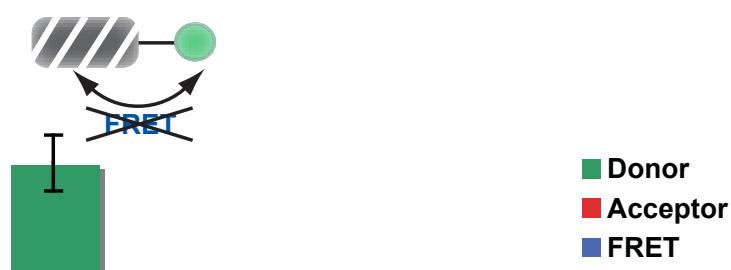
incubated with 5

Average intensities of cells microinjected with 3

Figure S8. Quantitative analysis of cells incubated with protein 5 for 1 hour. (A) Representative fluorescence images. Images were acquired on a confocal microscope and pseudo-colored green for the donor image $(\mathrm{Ex}=488 \mathrm{~nm}, \mathrm{Em}=525 \mathrm{~nm})$, red for the acceptor image $(\mathrm{Ex}=568 \mathrm{~nm}$, $\mathrm{Em}=620 \mathrm{~nm})$, and blue for the FRET image $(\mathrm{Ex}=488 \mathrm{~nm}, \mathrm{Em}=620 \mathrm{~nm}$, corrected for donor and acceptor cross-talk). Mean fluorescence intensities and their corresponding standard deviations were recorded in the nuclear compartment of each cells. (B) Relative and average fluorescence intensities recorded from 32 cells incubated with protein $\mathbf{5}$. The fluorescence intensities were normalized to the FRET emission and compared to the average intensities of 20 cells microinjected with protein $\mathbf{3}$. 


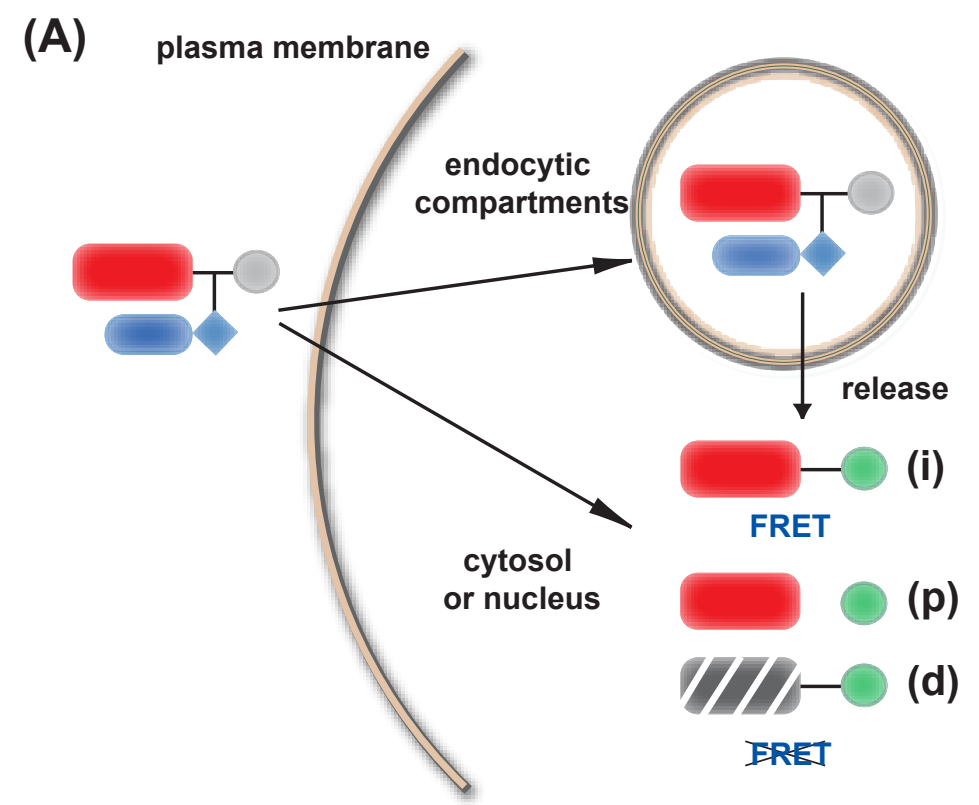

(B)

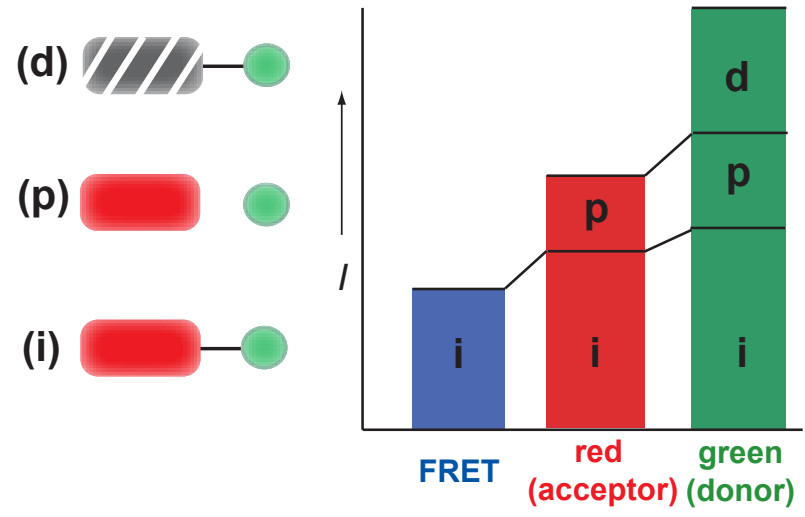

Figure S9: (A) Scheme illustrating the different populations of protein that can be expected in the cytosol or nucleus of cells after protein transduction, including intact (i), proteolytically cleaved ( $p)$, and degraded or unfolded (d) proteins.

(B) Expected fluorescence contributions of the cytosolic or nuclear protein populations. As illustrated, activation of the green fluorophore is independent from the biophysical state of the protein and donor fluorescence can be expected from (i), (p), and (d). Acceptor fluorescence is an intrinsic property of the folded protein and both (i) and (p) will contribute to this signal. Finally, the FRET signal is unique to the intact cytosolic or nuclear protein (i), therefore providing a specific signal for the successfully delivered protein. 